\title{
Clarifying "never events" and introducing "always events"
} Alan Lembitz* and Ted J Clarke

\author{
Address: Colorado Physician Insurance Company (COPIC), Headquarters, Denver, CO 80230, USA \\ Email: Alan Lembitz* - alembitz@copic.com; Ted J Clarke - tclarke@copic.com \\ * Corresponding author
}

Published: 3I December 2009

Patient Safety in Surgery 2009, 3:26 doi:10.1 186/1754-9493-3-26

This article is available from: http://www.pssjournal.com/content/3/I/26

(c) 2009 Lembitz and Clarke; licensee BioMed Central Ltd.

This is an Open Access article distributed under the terms of the Creative Commons Attribution License (http://creativecommons.org/licenses/by/2.0), which permits unrestricted use, distribution, and reproduction in any medium, provided the original work is properly cited.
Received: 17 December 2009

Accepted: 31 December 2009 
Table I: Serious reportable events ("never-events"), as defined by the National Quality Forum (NQF consensus report, update 2006; http://www.qualityforum.org)

I. Surgery performed on the wrong body part.

2. Surgery performed on the wrong patient.

3. Wrong surgical procedure performed on a patient.

4. Unintended retention of a foreign object in a patient after surgery or other procedure.

5. Intraoperative or immediate postoperative death in an ASA class I patient.

6. Patient death or serious disability associated with the use of contaminated drugs, devices, or biologics provided by the healthcare facility.

7. Patient death or serious disability associated with the use or function of a device in patient care in which the device is used or functions other than as intended.

8. Patient death or serious disability associated with intravascular air embolism that occurs while being cared for in a healthcare facility.

9. Infant discharged to the wrong person.

10. Patient death or serious disability associated with patient elopement (disappearance)

II. Patient suicide, or attempted suicide, resulting in serious disability while being cared for in a healthcare facility.

12. Patient death or serious disability associated with a medication error.

13. Patient death or serious disability associated with a haemolytic reaction due to the administration of ABO/HLA-incompatible blood or blood products.

14. Maternal death or serious disability associated with labor or delivery in a low-risk pregnancy while being cared for in a healthcare facility.

15. Patient death or serious disability associated with hypoglycaemia, the onset of which occurs while the patient is being cared for in a healthcare facility.

16. Death or serious disability (kernicterus) associated with failure to identify and treat hyperbilirubinemia in neonates.

17. Stage 3 or 4 pressure ulcers acquired after admission to a healthcare facility.

18. Patient death or serious disability due to spinal manipulative therapy.

19. Artificial insemination with wrong donor sperm or wrong egg.

20. Patient death or serious disability associated with an electric shock while being cared for in a healthcare facility.

21. Any incident in which a line designated for oxygen or other gas to be delivered to a patient contains the wrong gas or is contaminated with toxic substances.

22. Patient death or serious disability associated with a burn incurred from any source while being cared for in a healthcare facility.

23. Patient death or serious disability associated with a fall while being cared for in a healthcare facility.

24. Patient death or serious disability associated with the use of restraints or bedrails while being cared for in a healthcare facility.

25. Any instance of care ordered by or provided by someone impersonating a physician, nurse, pharmacist, or other licensed healthcare provider.

26. Abduction of a patient of any age.

27. Sexual assault on a patient within or on the grounds of a healthcare facility.

28. Death or significant injury of a patient or staff member resulting from a physical assault (i.e. battery) that occurs within or on the grounds of a healthcare facility. 
ative carries an extra psychological charge. Dr. Milstein also points to Kahneman and Tversky's Nobel prize winning research on "negative framing" which suggests that humans are more strongly inclined to take action when the actions in question are labeled so as to convey the loss avoided (rather than the benefit gained) and when the consequences of failing to act are mentally vivid [7].

\section{Liability concerns and negligence claims}

The biggest concern we face is the public confusion between the two lists, based on two distinct definitions by the NQF and CMS, respectively. Most, but not all, of the events on the NQF "never events" likely carry liability. While the amount of compensation may be questionable, few argue against the just compensation for injuries that result from never events. However, many of the non-reimbursable CMS "never events" are not completely preventable, even with the best practice of evidence-based treatment. We are concerned that patients experiencing complications listed as "non-reimbursable serious hospital-acquired conditions" will be inaccurately told that those "never events" are based on negligence or medical errors. It won't be long before we see trial lawyers advertise and openly solicit patients with conditions deemed "never events" or "non-reimbursable events".

Although many of the events listed by NQF and CMS are preventable, this is not always the case (Figure 1). We wish to emphasize and discuss some examples related to the controversy related to the preventability of some of the listed conditions:

\section{(I) Prevention of falls}

A recent editorial in the New England Journal of Medicine argues that the inclusion of "falls" on both lists is misguided[8]. According to Dr. Sharon Inouye (Harvard Medical School), there is currently no evidence that hospital falls "(..) can be consistently and effectively prevented through the application of evidence-based guidelines. (...) Their inclusion may have unintended consequences that may cause greater harm than the falls that the initiative is meant to prevent" [8].

Unintended consequences are likely to include a decrease in mobility, increase in use of physical restraints, and a tendency to focus on measures including new prevention devices. These measures can cause reallocation of resources from areas that might have greater impacts on patient safety. According to the editorial, "Falls are often the result not of medical errors but of disease, impairments, and appropriate uses of medications and other treatments. Falls and injuries can occur even when hospitals provide the best possible care" [8].

\section{(2) Postoperative infections and thromboembolic events}

It is known that certain orthopaedic procedures can result in the hospital-acquired conditions of postoperative infections and thromboembolic events. Neither compli- cation can ever be completely prevented. Arguments also exist that vigorous thromboprophylaxis in certain orthopaedic procedures can lead to an increased risk of delayed bleeding, wound healing problems, and postoperative infections. This notion has led the American Academy of Orthopaedic Surgeons (AAOS) to recommend different prophylaxis regimens compared to the evidence-based guidelines published by the American College of Chest Physicians (ACCP)[9].

We strongly feel that there is a need to officially clarify that falls, postoperative infections, and thromboembolic events are "non-reimbursable serious hospital-acquired conditions", but not "never events". Efforts certainly must be made to reduce either complication as much as possible via evidence-based assessment and treatment. Documentation of the informed consent process, and the risk/ benefit analysis underlying the clinical decision making processes are critical to patient understanding of potential complications and our ability to defend the care provided in the medicolegal setting.

\section{Strategies to reduce risk}

Strategies to improve the defensibility of care where appropriate, particularly those falling under the non-preventable adverse events list include:

- Pretreatment or pre-hospital documentation of underlying pre-existing conditions, particularly those involving infections, pressure sores, altered mental status, hyper-/hypoglycemia, and patients at high risk for venous thromboembolism.

- Hospital outcomes data with identification of care improvements directed at those complications - particularly hospital-acquired infections.

- Standardized and universally followed approaches to reduce wrong site/wrong patient surgery.

- Culture-changing training around communication, assertiveness, team training, and the use of briefings and debriefings, particularly in high-acuity patient care areas.

- The use of surgical checklists.

- Understanding and using clear language in policies and publications of the difference between the NQF "never events" and the CMS "non-reimbursable serious hospital-acquired conditions" to avoid claims of negligence.

\section{Introducing a positive approach towards patient safety: the "always events"}

"Never events" and non-reimbursable adverse events are framed in the negative and likely carry some "extra psy- 
1. "Never" and "No pay"

Events which overlap between the NQF and CMS definitions of "never events"

- Surgery on the wrong body part

- Surgery on the wrong patient

- Wrong surgery on a patient

- Foreign object left in patient after surgery

- Death/disability associated with intravascular air embolism

- Death/disability associated with incompatible blood

- Death/disability associated with hypoglycemia (HAC's include diabetic ketoacidosis, nonketotic hyperosmolar coma, hypoglycemic coma, secondary diabetes with

ketoacidosis, secondary diabetes with hyperosmolarity)

- Stage 3 or 4 pressure ulcers after admission

- Death/disability associated with electric shock

- Death/disability associated with a burn incurred within facility

- Death/disability associated with a fall within facility

2. "Never"

Events which should never happen according to the NQF, but are not listed on the CMS "never events").

- Postoperative death in a healthy patient

- Implantation of wrong egg

- Death/disability associated with use of contaminated drugs, devices, or biologics

- Death/disability associated with use of device other than as intended

- Infant discharged to wrong person

- Death/disability due to patient elopement

- Patient suicide or attempted suicide resulting in disability

- Death/disability associated with medication error

- Maternal death/disability with low risk delivery

- Death/disability associated with hyperbilirubinemia in neonates

- Death/disability due to spinal manipulative therapy

- Incident due to wrong oxygen or other gas

- Death/disability associated with use of restraints within facility

- Impersonating a health care provider (i.e., physician, nurse)

- Abduction of a patient

- Sexual assault of a patient within or on facility grounds

- Death/disability resulting from physical assault within/on facility grounds

\section{3. "No pay"}

The list of controversy: Adverse events which are classified by the CMS as nonreimbursable "never events", but lack the according definition by the NQF.

- Catheter-associated urinary tract infection

- Vascular catheter-associated infection

- Surgical site infection following coronary artery bypass graft (CABG) - mediastinitis

- Surgical site infection following bariatric surgery (laparoscopic gastric bypass, gastroenterostomy, laproscopic gastric restrictive surgery)

- Surgical site infection following orthopedic procedures (spine, neck, shoulder, elbow)

- Deep vein thrombosis (DVT)/pulmonary embolism (PE) in total knee replacement and hip replacement

Figure I

Comparison of "never events", as defined by the NQF ("serious reportable events") versus CMS ("non-reimbursable serious hospital-acquired conditions"). 
chological charge", as mentioned above. Our concept of the "always events" represents a positive affirming behavior that can motivate us to improve patient safety and promote better outcomes. Some basic examples of "always events" include:

- Including patient identification by more than one source.

- Mandatory "readbacks" of verbal orders for highalert medications.

- Disclosure of adverse outcomes and transparency with patients and families.

- Medication error reduction strategies.

- Surgical time-out.

- Anesthesia monitoring that is appropriate for the level of sedation.

- Tracking of critical imaging, lab and pathology results.

- Making critical information available at handoffs or transitions in care.

Standardization and validation of "always events" may represent the basis for a positive long-term culture of patient safety to be passed on to the next generation of health care providers.

\section{Competing interests}

Both authors are affiliated with the Colorado Physician Insurance Company (COPIC). The author declares no other competing interests with regard to this manuscript.

\section{Authors' contributions}

Both authors contributed equally in the design and writing of this editorial.

\section{Acknowledgements}

This article represents an invited guest editorial based on a previously published article in the COPIC risk management newsletter (COPISCOPE

2009). Reproduced in modified version with permission.

\section{References}

I. Stahel PF, Fakler JKM, Smith WR, Clarke TJ, Mehler PS: Patient safety in surgery: what lessons can we learn from the current US standards? Periop Med 2009, I (I):34-43.

2. Michaels RK, Makary MA, Dahab Y, et al.: Achieving the National Quality Forum's "Never Events": prevention of wrong site, wrong procedure, and wrong patient operations. Ann Surg 2007, 245(4):526-532.

3. Stahel PF, Sabel AL, Victoroff MS, Varnell J, Lembitz A, Boyle DJ, Clarke TJ, Smith WR, Mehler PS: Wrong site and wrong patient surgery in the era of the "Universal Protocol": Analysis of a prospective database of physician self-reported occurrences. Arch Surg 2010 in press.

4. Gallagher TH, Studdert D, Levinson W: Disclosing harmful medical errors to patients. N Engl J Med 2007, 356:27| 3-9.

5. Murphy JG, Stee L, McEvoy MT, Oshiro J: Journal reporting of medical errors: the wisdom of Solomon, the bravery of Achilles, and the foolishness of Pan. Chest 2007, 13 I:890-6.

6. Stahel PF, Flierl MA, Smith WR, Morgan SJ, Victoroff MS, Clarke TJ, Sabel AL, Mehler PS: Disclosure of surgical complications - a double-edged sword? Am J Med Qual 2010 in press.

7. Milstein A: Ending extra payment for "never events" stronger incentives for patients' safety. N Engl J Med 2009, 360:2388-90.

8. Inouye SK, Brown CJ, Tinetti ME: Medicare nonpayment, hospital falls, and unintended consequences. N Engl J Med 2009, 360:2390-3.

9. Geerts WH, Bergqvist D, Pineo GF, Heit JA, Samama CM, Lassen MR, Colwell CW: Prevention of venous thromboembolism: American College of Chest Physicians' evidence-based clinical practice guidelines. Chest 2008, 133:38I-453.
Publish with Biomed Central and every scientist can read your work free of charge

"BioMed Central will be the most significant development for disseminating the results of biomedical research in our lifetime. "

Sir Paul Nurse, Cancer Research UK

Your research papers will be:

- available free of charge to the entire biomedical community

- peer reviewed and published immediately upon acceptance

- cited in PubMed and archived on PubMed Central

- yours - you keep the copyright 